\title{
Cisplatin-Etoposide Induced Myocardial Infarction in a Patient with Small Cell Carcinoma
}

\author{
Srija Katta ${ }^{1}$, Sheetal Chauhan ${ }^{2}$, Ajit Singh ${ }^{3 *}$, Prasad N. Shetty ${ }^{3}$, Hashir Kareem ${ }^{3}$, Tom Devasia ${ }^{3}$ \\ ${ }^{1}$ Department of Pharmacy Practice, St. Peter's Institute of Pharmaceutical Sciences, Hanamkonda, Telangana-506001, India. \\ ${ }^{2}$ Department of Pharmacology, Melaka Manipal Medical College, Manipal Academy of Higher Education, Manipal-576104, India. \\ ${ }^{3}$ Department of Cardiology, Kasturba Medical College and Hospital, Manipal Academy of Higher Education, Manipal-576104, India.
}

\section{ARTICLE INFO \\ Article history: \\ Received on: 23/12/2017 \\ Accepted on: 21/02/2018 \\ Available online: 30/05/2018}

Key words:

Chemotherapy-

induced Cardiotoxicity,

Anthracycline,

Bronchogenic carcinoma,

Antineoplastic drugs.

\begin{abstract}
Various drugs used to treat cancer cause hemodynamic/physiological changes in various organs of the body. Cardiotoxicity is one of the major side effects. Cardiotoxicity associated with chemotherapy can range from asymptomatic sub-clinical echocardiographic changes to life-threatening events like congestive heart failure or acute coronary syndrome. We present a case of cisplatin and etoposide-induced myocardial infarction (MI) during the second cycle of chemotherapy for bronchogenic carcinoma of the left lung (small cell carcinoma). The patient developed troponin positive NSTEMI and was managed conservatively. Here, the possible mechanism of action and the importance of cardiac screening while using cisplatin and etoposide are discussed.
\end{abstract}

\section{INTRODUCTION}

Life is harsh for cancer survivors and also on those who are on cancer chemotherapy. They have a list of problems hitting them along with cancer and its treatment. Chemotherapy agents have a lot of side effects and affect various organs/ systems inside the body physiologically or hemodynamically. The heart is the most affected organ by anthracyclines and other cancer treating drugs. Life-threatening arrhythmias, left ventricle dysfunction with/without clinical heart failure and acute coronary syndrome may be seen during the chemotherapy or within 2-4 weeks of termination of chemotherapy (Berardi et al., 2013). Among cardiovascular events caused by chemotherapy agents, the incidence of myocardial infarction (MI) is $0.24 \%$ (Dieckmann et al., 2010). Here we discuss a male patient who developed myocardial infarction (MI) during his second cycle of

\section{"Corresponding Author}

Ajit Singh, Department of Cardiology, Kasturba Medical College and Hospital, Manipal Academy of Higher Education, Manipal-576104, India.E-mail:ajitjsingh.mcops@gmail.com chemotherapy with cisplatin and etoposide for small cell cancer of the left lung. Only a few cases of MI have been reported with this combination thus far.

Our aim to present the case is to understand the possible mechanism and course of treatment for such cases.

\section{CASE REPORT}

A 62-year-old male was admitted to the medical oncology department of Kasturba Medical College and Hospital for his second cycle of chemotherapy for small cell carcinoma left lung. After his second round of chemotherapy for three days (Cisplatin $80 \mathrm{mg} / \mathrm{m}^{2} \mathrm{IV}$ on day one plus etoposide $100 \mathrm{mg} / \mathrm{m}^{2} \mathrm{IV}$ on days 1-3), the patient developed sudden onset of angina and dyspnea.

He had a history of hypertension (HTN) for 20 years and was being treated with telmisartan $40 \mathrm{mg}$ and amlodipine 5 mg PO once daily. He was diagnosed to have chronic obstructive pulmonary disease (COPD) 2 years back, for which he was admitted twice in the last two years. He had an exacerbation of COPD three months back, during which he was diagnosed 
to have bronchogenic carcinoma of the left lung (small cell carcinoma). Contrast-enhanced computed tomography (CECT) and bronchoscopic biopsy confirmed the diagnosis (Figure 1). Positron emission tomography (PET) showed a large left perihilar lung mass, a large cavity with few calcific specs, metastatic prevascular, per-cardinal, subcarinal and left hilar nodes. The patient was treated with chemotherapy (Cisplatin $200 \mathrm{mg} / \mathrm{m}^{2} \mathrm{IV}$ on day one plus etoposide $100 \mathrm{mg} / \mathrm{m}^{2} \mathrm{IV}$ on days 1-3). The patient was a chronic smoker for 25 years (1 pack of cigarettes daily; 20-30 cigarettes/pack).

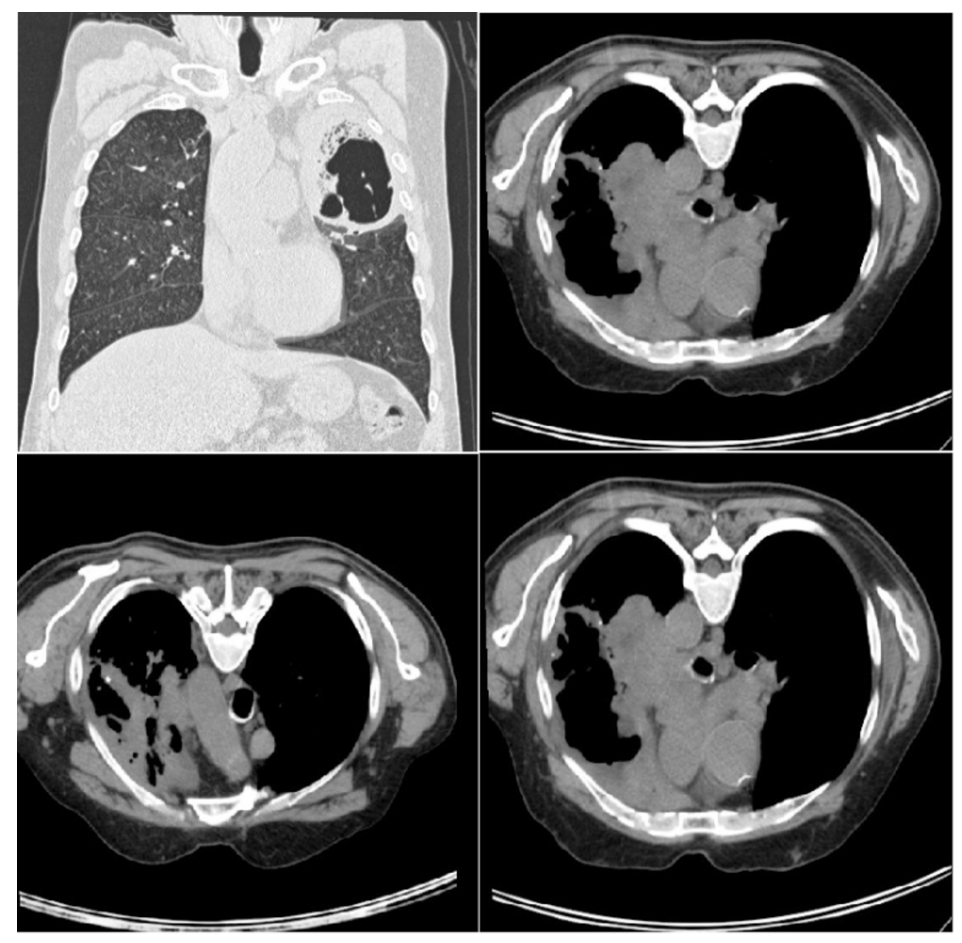

Fig. 1: CECT showed large left perihilar lung mass, a large cavity with few calcific specs, metastatic prevascular, per-cardinal, subcarinal and left hilar nodes suggesting bronchogenic carcinoma.

Investigations were done, and electrocardiogram (ECG) showed $\mathrm{T}$ inversion in lateral leads (Figure 2). Echocardiogram revealed globally hypokinetic left ventricle (LV), mild LV systolic dysfunction with an ejection fraction of $45 \%$, mild mitral regurgitation $(\mathrm{MR})$, and restrictive filling pattern of LV. As the troponin levels were raised $(0.9 \mathrm{ng} / \mathrm{mL})$, the patient was diagnosed to have acute coronary syndrome, with NSTEMI. Coronary angiogram (CAG) showed a single vessel disease (SVD) RAMUS ostioproximal complete occlusion, filling retrograde from grade II collaterals) and branch vessel disease (Diagonal total occlusion, filling retrogradely from grade II collaterals).

Development of pneumonia complicated his MI during hospitalization which was confirmed by x-ray chest (Figure 3) and other blood tests. CT thorax confirmed lung abscess. Sputum culture was positive for Pseudomonas aeruginosa for which he was given antibiotics (piperacillin and tazobactam combination, metronidazole and amikacin) based on sensitivity test.

The patient was stable and advised to continue optimized medical therapy without any intervention at this stage. Antiplatelet medications including aspirin $75 \mathrm{mg}$ PO daily, clopidogrel $75 \mathrm{mg}$ PO daily along with atorvastatin $10 \mathrm{mg}$ PO daily and nitrates were continued. Telmisartan and amlodipine were discontinued and prazosin $2.5 \mathrm{mg}$ PO twice daily was started for blood pressure control and heart failure therapy. The patient is doing well at one month follow up.

\section{DISCUSSION}

The majority of the chemotherapy drugs cause cardiotoxicity; anthracycline and trastuzumab have a significant effect on the heart (Ibraheem et al., 2016). Cardiotoxicity associated with chemotherapy can range from asymptomatic sub-clinical echocardiographic changes to life-threatening events like decompensated congestive heart failure or acute coronary syndrome (Berardi et al., 2013). Chemotherapy drugs like tyrosine kinase inhibitors (TKIs), anthracyclines, alkylating agents, and interferon-alpha are associated with myocardial dysfunction, mainly left ventricular dysfunction (LVD) (Swain et al., 2003). In this case, the patient presented with ACS (NSTEMI) during the second cycle of chemotherapy. A similar case was reported in a male patient with testicular seminoma during the third cycle of bleomycin, etoposide and cisplatin regimen, the patient had symptoms of ACS but with ST elevation (Lee et al., 1990). Another patient with small cell carcinoma also presented with breathlessness, chest pain, severe diaphoresis and high-grade fever for 2 days after the second cycle of etoposide and cisplatin combination chemotherapy and 31 fractions of radiotherapy; this combination chemotherapy caused an episode of vasospastic angina leading to acute myocardial infarction and STEMI (Hanchate, 2017). Two patients with testicular cancer who were treated with combination therapy of bleomycin, etoposide, and cisplatin were reported to develop sudden thoracic pain and were 
both diagnosed with acute STEMI (Rao et al., 2015). The patient we are presenting here was a chronic smoker and hypertensive. These conditions also could contribute to ACS and MI (Lapubula et al., 2011; Kawano et al., 2013). The possible risk factors for anthracycline-associated cardiotoxicity are age, cumulative dose, combination therapy, mediastinal radiotherapy, concurrent cardiovascular diseases, gender, chromosomal abnormalities and administrative schedules (Volkova and Russell, 2012). In this case, age and combination therapy might have been the risk factors for cardiotoxicity. The mechanism of action of cisplatin-induced MI, in this case, maybe type 1 cardiotoxicity caused by cardiomyocyte death, either through necrosis or apoptosis (Berardi et al., 2013). This necrosis or apoptosis may be due to multiple forms of cell injury by free radical production, altered nucleic acid biology by intercalation into DNA and modulating intracellular signaling (Swain et al., 2003). Damage to vessels and heart muscles can be divided into two types, damage type I and damage type II. Damage type I is an irreversible injury because of the cytostatic effect of traditional antineoplastic drugs and associated with myocytic death, damage type II reversibly altered signaling pathways due to newer anticancer drugs associated with myocardial stunning or cellular hibernation (Geisberg and Sawyer, 2010).

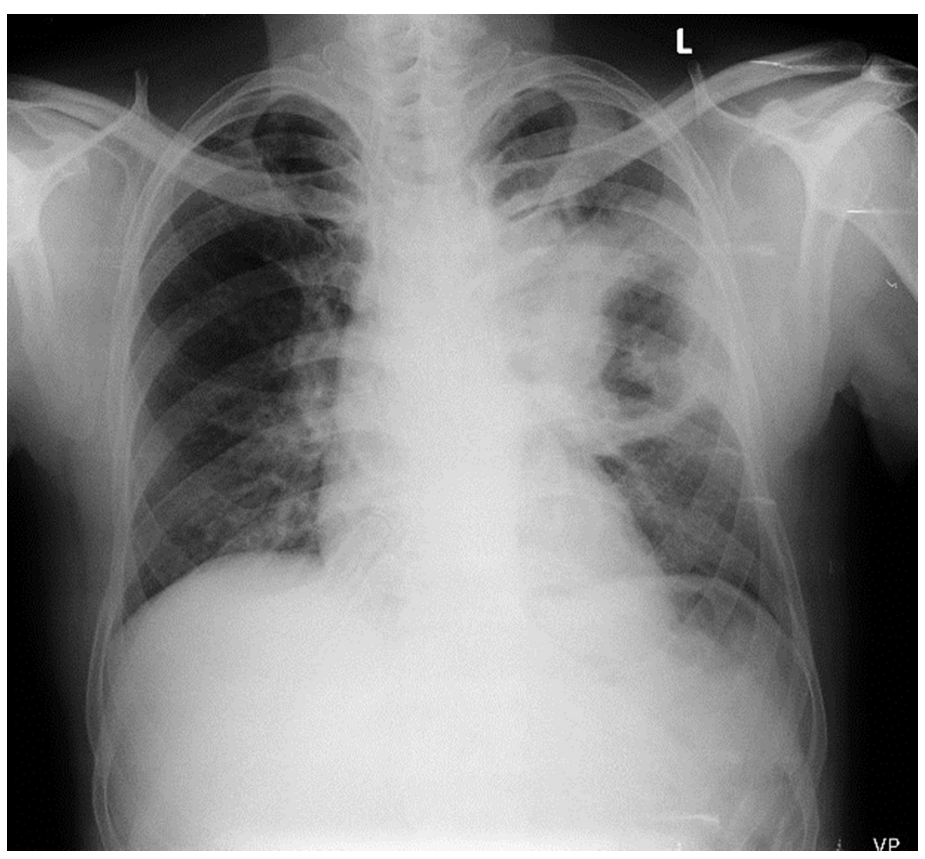

Fig. 2: Electrocardiogram showing $T$ inversion in lateral leads.

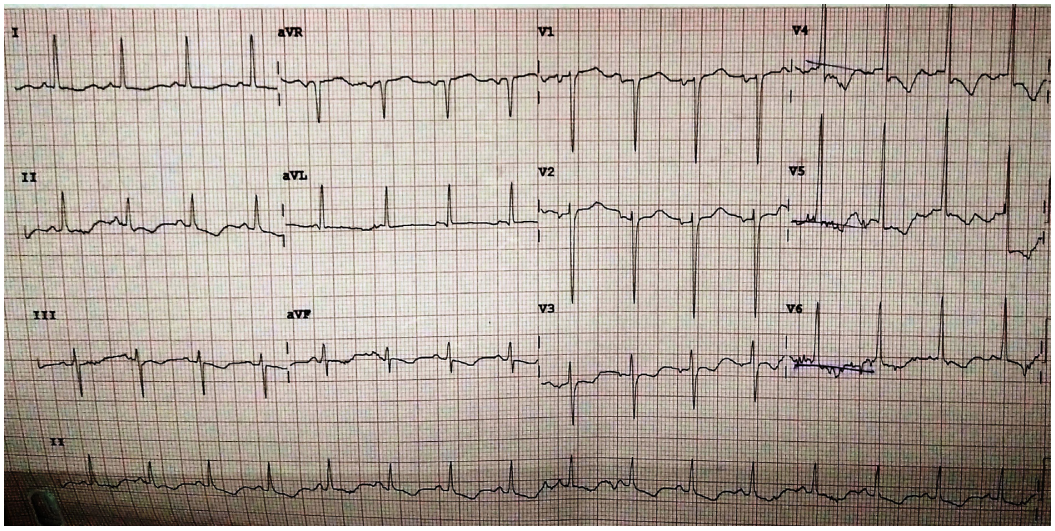

Fig. 3: Chest X-ray suggested the pneumonia patches.

The release of inflammatory mediators probably destabilizes the vulnerable plaque causing total occlusion and NSTEMI. However, hypokinesia in ECHO suggests that additional mechanism including apoptosis or myocardial stunning could have contributed to myocardial dysfunction and HF in the background of HTN and smoking for this patient more than 60 years of age.

\section{CONCLUSION}

Etoposide-cisplatin combination can cause the lifethreatening events like MI. The oncologist should be careful at the time of therapy selection; therapy with less cardiac impact. Cardiac parameters should be evaluated at various time points; before starting the chemotherapy, during and after completion of chemotherapy to assess the physiological/hemodynamic changes 
in the heart. A collaborative approach between cardiologist and oncologist can improve the therapeutic outcomes in such patients.

\section{INFORMED CONSENT}

Informed consent was taken from the patient before examination and publication.

\section{REFERENCE}

Berardi R, Caramanti M, Savini A, Chiorrini S, Pierantoni C, Onofri A, et al. State of the art for cardiotoxicity due to chemotherapy and to targeted therapies: A literature review. Critical Reviews in Oncology/ Hematology, 2013; 88(1):75-86.

Dieckmann K, Gerl A, Witt J, Hartmann J. Myocardial infarction and other major vascular events during chemotherapy for testicular cancer. Annals of Oncology, 2010; 21(8):1607-1611.

Geisberg C, Sawyer D. Mechanisms of Anthracycline Cardiotoxicity and Strategies to Decrease Cardiac Damage. Current Hypertension Reports, 2010; 12(6):404-410.

Hanchate LP, Sharma SR, Madyalkar S. Cisplatin Induced Acute Myocardial Infarction and Dyslipidemia. Journal of clinical and diagnostic research. JCDR, 2017; 11(6):OD05.

Ibraheem A, Stankowski-Drengler T, Gbolahan O, Engel J, Onitilo A. Chemotherapy-induced cardiotoxicity in breast cancer patients. Breast Cancer Management, 2016; 5(1):31-41.

Kawano N, Yamaguchi K, Niki $T$, Yamamoto $T$, Iwase $T$, Taketani Y, et al. Two cases of acute myocardial infarction during combined chemotherapy in young patients with testicular cancer. Journal of Cardiology Cases, 2013; 7(6):e176-e180.

Lapu-Bula R, Onwuanyi A, Bielo MV, Deffer O, Quarshie A, Alema-Mensah E, et al. Risk factors for acute non-ST-segment elevation myocardial infarction in a population sample of predominantly African American patients with chest pain and normal coronary arteries. Ethnicity \& disease, 2011; 21(4):421.

Lee K, Lee J, Kim S. Electrocardiographic Changes Simulating Acute Myocardial Infarction or Ischemia Associated with Combination Chemotherapy with Etoposide, Cisplatin, and 5-Fluorouracil. The Korean Journal of Internal Medicine, 1990; 5(2):112-118.

Rao A, Kumar R, Narayanan G. A rare case of cisplatin-induced acute myocardial infarction in a patient receiving chemoradiation for lung cancer. Journal of Cancer Research and Therapeutics, 2015; 11(4):983.

Swain SM, Whaley FS, Ewer MS. Congestive heart failure in patients treated with doxorubicin: a retrospective analysis of three trials. Cancer, 2003; 97:2869-79.

Volkova M, Russell R. Anthracycline Cardiotoxicity: Prevalence, Pathogenesis and Treatment. Current Cardiology Reviews, 2012; 7(4):214220.

How to cite this article:

Katta S, Chauhan S, Singh A, Shetty PN, Kareem H, Devasia T. Cisplatin-Etoposide Induced Myocardial Infarction in a Patient with Small Cell Carcinoma. J App Pharm Sci, 2018; 8(05): 139142. 